\title{
The next frontier in critical care guidelines: rapid and trustworthy recommendations
}

\author{
Reed A. C. Siemieniuk, MD • Gordon H. Guyatt, MD, MSc
}

Received: 15 December 2016/Revised: 25 December 2016/Accepted: 11 April 2017/Published online: 11 May 2017

(c) Canadian Anesthesiologists' Society 2017

Optimal clinical care requires application of the best available evidence to patient care decisions. ${ }^{1}$ Trustworthy clinical practice guidelines synthesize the available evidence and provide suggestions for front-line clinicians.

Historically, guideline panels take months to, most commonly, years to process the evidence and produce their recommendations. Many conventional guidelines are outdated by the time of publication, and almost all are outdated before the panel produces an update. In the hiatus between the publication of new evidence and its inclusion in a guideline, clinicians and patients are left wondering whether the new evidence should change practiceassuming they are even aware of the new evidence. As a result, many patients are at risk of receiving inadequate or inappropriate care.

The Canadian Critical Care Society (CCCS) and the Scandinavian Society of Anaesthesiology and Intensive Care (SSAI) have taken a leap forward in guideline creation. The accompanying guidelines are the first in a series of rapid recommendations. ${ }^{2,3}$ The project is part of a collaborative initiative with the MAGIC Project (www. magicproject.org) called WikiRecs-"Wiki" means

R. A. C. Siemieniuk, MD ( $₫) \cdot$ G. H. Guyatt, MD, MSc Department of Health Research Methods, Evidence, and Impact, McMaster University, 1280 Main St W, Hamilton, ON L8S 4L8, Canada

e-mail: reed.siemieniuk@medportal.ca

R. A. C. Siemieniuk, MD

Department of Medicine, University of Toronto, Toronto, ON, Canada

G. H. Guyatt, MD, MSc

Department of Medicine, McMaster University, Hamilton, ON, Canada "rapid" in Hawaiian-modelled after the new BMJ series, "Rapid Recommendations". 4,5

A recently published randomized trial addressed an important topic, i.e., optimal blood pressure targets in critically ill patients. ${ }^{6}$ In response, the leaders of the Critical Care-WikiRecs group developed a rapid clinical practice guideline by following the WikiRecs process. ${ }^{4}$

The process starts when the WikiRecs team identifies a new study that might initiate a change in practice. A guideline panel then convenes to determine which populations, outcomes, and subgroups to consider. A semi-independent systematic review team then quickly synthesizes all the evidence for all patient-important outcomes and subgroups identified by the panel. The panel considers the evidence in the context of patient values and preferences and makes a recommendationeither strong or weak-in favour of the intervention or the comparator.

Notwithstanding the rapidity of the process, the recommendations adhere to the stringent Institute of Medicine standards ${ }^{7}$ - standards that most conventional guidelines fail to achieve. ${ }^{8} \mathrm{~A}$ standard that WikiRecs meets but is often neglected by conventional guideline panels includes participation by a complete spectrum of stakeholders, including critical care specialists, a surgeon, a nurse, methodologists, and a former patient. The patient panel member was key in identifying all of the most patient-important outcomes, including long-term quality of life, an outcome not captured in any relevant randomized trials (and thus representing a gap in current knowledge).

The recommendations, based on systematic reviews using the GRADE (Grading of Recommendations Assessment, Development and Evaluation) approach, ${ }^{9}$ include a formal assessment of the certainty and magnitude of the effects on each outcome. ${ }^{10,11}$ The 
authors use the GRADE evidence profiles which utilize formats tested in randomized trials ${ }^{12,13}$ in a multilayered format available on all devices electronically through MagicApp (www.magicapp.org). Moreover, the guideline panel considered and presented all practical issues, together with the key implementation issues, including nursing resources-a subject that guideline panels do not always consider.

Although the issue of conflict of interest on guideline panels has received considerable attention ${ }^{14}$ and authors have suggested solutions, ${ }^{15}$ it is not uncommon for guideline panels to continue including panellists with serious conflicts of interest. ${ }^{8}$ In this case, any persons with any potential financial conflicts were excluded.

Managing intellectual conflicts is more difficult to navigate because the leading content experts have often participated in research or have made public statements that are likely to leave them attached to certain viewpoints. In this case, a panel member (F.L.) was the first author of a randomized trial on blood pressure targets in shock. ${ }^{6}$ The panel appears otherwise free of conflicts.

The panel made a weak recommendation for a lower (mean arterial pressure $=60-70 \mathrm{mmHg}$ ) rather than a higher blood pressure target. ${ }^{3}$ The rationale is clearly articulated, i.e., the panel judged that most patients would prefer to avoid a possible increased risk of myocardial infarction (very low certainty) and arrhythmias (moderate certainty) in the absence of evidence that a higher target reduces mortality (low certainty) or has any other benefits. Further, the panel recognized the substantial resources required to implement a higher blood pressure targetmostly due to increased nursing demands. The recommendation was weak rather than strong because of the low-quality evidence available regarding outcomes most important to patients, i.e., mortality and quality of life.

For vasopressors in early traumatic shock, the panel considered the trade-offs between risks and benefits too close and the uncertainty too great to make a recommendation. ${ }^{2}$ One could question the panel's decision not to make a recommendation: it is unlikely that anyone will have perused the evidence as thoroughly as the panel, and clinicians need guidance from those who have. The authors do, however, provide a GRADE-style evidence summary in the publication and in an interactive multilayered format on MagicApp—a summary that clinicians can use to arrive at their own conclusions.

The goal of the WikiRecs project is simple, i.e., to move evidence more quickly into practice using a process that clinicians can trust. These first CCCS and SSAI co-led rapid recommendations demonstrate that this is possible. The success of the project requires buy-in from front-line clinicians and a willingness to adapt to criticism. Most importantly, the success of rapid recommendations depends on a commitment to adhere stringently to standards for trustworthiness. It is possible that these CCCS/SSAI recommendations, rapid and trustworthy, represent an early sign of broader change within the guideline industry towards more collaborative, trustworthy, timely, and user-friendly recommendations.

\section{Prochaine étape pour les directives en soins intensifs : des recommandations rapides et dignes de confiance}

Pour offrir des soins cliniques optimaux, il faut appliquer les meilleures données probantes disponibles lorsqu'on prend des décisions en matière de soins aux patients. ${ }^{1}$ Pour être dignes de confiance, les directives de pratique clinique doivent résumer les données probantes disponibles et proposer des solutions aux cliniciens de première ligne.

Historiquement, les panels en charge de rédiger les directives prennent des mois, même des années, à examiner les données probantes et émettre leurs recommandations. De nombreux guides d'exercice conventionnels ne sont déjà plus en ligne avec les dernières données probantes au moment de leur publication, et la vaste majorité de ces guides sont surannés lorsque le panel en charge en propose enfin une mise à jour. Entre le moment de publication de nouvelles données probantes et leur inclusion dans une directive, cliniciens et patients se demandent bien souvent si ces nouvelles données devraient avoir un impact sur la pratique - en prenant pour acquis que ces individus aient connaissance de ces nouvelles données. Par conséquent, de nombreux patients courent le risque de recevoir des soins inadéquats ou inadaptés.

La Société canadienne de soins intensifs (CCCS) et la Société scandinave d'anesthésiologie et de soins intensifs (Scandinavian Society of Anaesthesiology and Intensive Care - SSAI) ont fait un véritable bond en avant dans la création de directives. Les directives qui découlent de cette initiative sont les premières d'une série de recommandations rapides. ${ }^{2,3}$ Le projet s'inscrit dans le cadre d'un projet réalisé en collaboration avec l'organisme MAGIC (www.magicproject.org), intitulé WikiRecs «wiki » signifie « rapide » en hawaïen -, qui s'est inspiré du modèle lancé par la nouvelle série de BMJ, les « $B M J$ Rapid Recommendations » ou « Recommandations rapides du BMJ ». 4,5 
Une étude randomisée récemment publiée a abordé un sujet important : les cibles de tension artérielle optimales chez les patients en état critique. ${ }^{6}$ Suite à cette étude, les chefs de file du groupe des Soins intensifs de WikiRecs ont mis ont point une directive de pratique clinique rapide selon le processus des WikiRecs. ${ }^{4}$

Le processus débute lorsque l'équipe des WikiRecs identifie une nouvelle étude qui pourrait modifier la pratique. Un panel de rédaction de directive se réunit alors et détermine de quelles populations, de quels critères d'évaluation et de quels sous-groupes tenir compte. Un compte rendu méthodique semi-indépendant résume ensuite rapidement toutes les données probantes concernant tous les critères d'évaluation importants pour les patients et tous les sous-groupes préalablement identifiés par le panel. Le panel étudie les données probantes selon les valeurs et les préférences des patients et émet une recommandation, forte ou faible, en faveur de l'intervention ou du comparateur.

Hormis la rapidité du processus, ces recommandations respectent les normes rigoureuses de l'Institute of Medicine américain $^{7}$ - des normes que la plupart des guides d'exercice conventionnels ont de la peine à respecter. ${ }^{8}$ Une des normes respectées par WikiRecs, souvent négligée par d'autres, est la participation d'un éventail complet d'intervenants, y compris des spécialistes des soins intensifs, un chirurgien, une infirmière, des méthodologues et un ancien patient. Le patient membre du panel a joué un rôle essentiel dans l'identification de tous les critères et pronostics importants aux yeux des patients, notamment la qualité de vie à long terme, un critère non évalué par les études randomisées pertinentes (mettant ainsi le doigt sur une lacune majeure des connaissances actuelles).

Les recommandations, fondées sur des comptes rendus méthodiques utilisant l'approche dite GRADE (pour Grading of Recommendations, Assessment, Development and Evaluation - soit Classification des recommandations, de l'analyse, de la mise au point et de l'évaluation), ${ }^{9}$ comprennent une évaluation formelle du degré de certitude et de l'ampleur des effets sur chaque critère d'évaluation. ${ }^{10,11}$ Les auteurs utilisent les profils de données probantes de GRADE, lesquels se fondent sur des formats testés dans des études randomisées ${ }^{12,13}$ dans un format multicouche disponible électroniquement sur tous les dispositifs grâce à l'application MagicApp (www. magicapp.org). Le panel a également tenu compte de et présente toutes les questions pratiques, notamment les principaux défis d'implantation (y compris les ressources en personnel infirmier - une donnée souvent oubliée par les panels responsables d'émettre des recommandations).

Bien que la question des conflits d'intérêt au sein des panels d'élaboration de recommandations ait fait l'objet de beaucoup d'attention ${ }^{14}$ et que plusieurs auteurs aient suggéré des solutions possibles, ${ }^{15}$ il est malheureusement encore fréquent que certains panélistes entretiennent d'importants conflits d'intérêt. ${ }^{8}$ Dans le cas du panel dont il est question ici, toute personne présentant un conflit d'intérêt potentiel d'ordre financier a été exclue.

La gestion des conflits intellectuels est plus difficile à naviguer parce que les experts d'une question donnée ont souvent pris part à des recherches, ou fait des déclarations publiques qui pourraient probablement les rattacher à certaines opinions. Dans le cas que nous présentons ici, un membre du panel (F.L.) est le premier auteur d'une étude randomisée sur les cibles de tension artérielle en cas de choc. ${ }^{6}$ Le panel semble autrement n'entretenir aucun conflit d'intérêt.

Le panel a émis une recommandation faible pour une cible de tension artérielle plus basse (tension artérielle moyenne $=60-70 \mathrm{mmHg}$ ) plutôt qu'une cible plus élevée. ${ }^{3}$ La raison est clairement expliquée : le panel a estimé que la plupart des patients préfèreraient éviter un risque potentiellement accru d'infarctus du myocarde (certitude très basse) et d'arythmie (certitude modérée) en l'absence de données probantes soutenant qu'une cible plus élevée réduirait la mortalité (certitude basse) ou aurait tout autre bienfait. En outre, le panel a reconnu l'ampleur des ressources nécessaires à la mise en œuvre d'une cible de tension artérielle plus élevée - principalement en raison des exigences plus grandes ainsi imposées au personnel infirmier. La recommandation était faible plutôt que forte en raison de la qualité médiocre des données probantes disponibles concernant les critères les plus importants pour les patients - soit la mortalité et la qualité de vie.

En ce qui touche à l'administration précoce de vasopresseurs après un choc traumatique, le panel a considéré que le compromis entre risques et bienfaits était trop fragile et que l'incertitude était trop grande pour émettre une recommandation, quelle qu'elle soit. ${ }^{2}$ On peut certes remettre en question la décision du panel, mais il est peu probable que quiconque ait examiné les données probantes avec autant d'attention et de soin que le panel, et les cliniciens ont besoin de conseils de personnes qui ont fait ce travail. Les auteurs fournissent toutefois un résumé des données probantes de style GRADE dans leur publication et dans un format multicouche interactif sur MagicApp, et les cliniciens peuvent utiliser ce résumé pour parvenir à leurs propres conclusions.

L'objectif du projet WikiRecs est simple : faire que les données probantes soient appliquées plus rapidement à la pratique grâce à un processus auquel les cliniciens peuvent se fier. Les premières recommandations rapides codirigées par le CCCS et la SSAI démontrent qu'une telle démarche est possible. Le succès du projet dépend du soutien des médecins de première ligne et d'une ouverture d'esprit 
pour pouvoir s'adapter aux critiques. Mais le succès des recommandations rapides dépend encore plus d'un engagement à respecter de façon rigoureuse des normes de fiabilité. Peut-être que ces recommandations des CCCS et SSAI, rapides et fiables, constitueront un signe avantcoureur d'un changement plus important encore au sein de l'industrie des directives, vers l'élaboration de recommandations plus collaboratives, dignes de confiance, publiées en temps opportun et faciles à utiliser pour le clinicien.

Disclosures Drs Reed A.C. Siemieniuk and Gordon H. Guyatt are members of the GRADE Working Group and MAGIC non-profit organization (Making GRADE the Irresistible Choice). The MAGIC organization and the authors are leāders of the WikiRecs and Rapid Recommendation projects. Drs Reed A.C. Siemieniuk and Gordon H. Guyatt are co-authors of the systematic reviews that informed the guidelines.

Conflicts of interest None declared.

Funding There are no financial conflicts of interest.

Editorial responsibility This submission was handled by Dr. Hilary P. Grocott, Editor-in-Chief, Canadian Journal of Anesthesia.

Déclarations Les Drs Reed A.C. Siemieniuk et Gordon H. Guyatt sont membres du groupe de travail GRADE et de l'organisme à but non lucratif MAGIC (Making GRADE the Irresistible Choice - Faire de GRADE le choix irrésistible). L'organisme MAGIC et les auteurs de cet éditorial sont à la tête des projets WikiRecs et Rapid Recommendation (recommandations rapides). Les Drs Reed A.C. Siemieniuk et Gordon H. Guyatt sont les co-auteurs des comptes rendus méthodiques qui ont permis d'élaborer ces directives.

\section{Conflit d'intérêt Aucun.}

Responsabilité éditoriale Cet article a été traité par Dr Hilary P. Grocott, rédacteur en chef, Journal canadien d'anesthésie.

Financement Il n'y a aucun conflit d'intérêt financier.

\section{References}

1. Evidence-Based Medicine Working Group. Evidence-based medicine. A new approach to teaching the practice of medicine. Jama 1992; 268: 2420-5.
2. Rochwerg B, Hylands M, Moller M, et al. CCCS-SSAI WikiRecs Clinical Practice Guideline: vasopressors in early traumatic shock. Can J Anesth 2017; 64: this issue. DOI: 10.1007/ s12630-017-0877-1

3. Rochwerg B, Hylands $M$, Moller M, et al. CCCS-SSAI WikiRecs Clinical Practice Guideline: vasopressor blood pressure targets in critically ill adults with hypotension. Can J Anesth 2017; 64: this issue. DOI: $10.1007 / \mathrm{s} 12630-017-0878-0$

4. Siemieniuk RA, Agoritsas T, Macdonald H, Guyatt GH, Brandt L, Vandvik $P O$. Introduction to BMJ rapid recommendations. BMJ 2016; 354: i5191.

5. Vandvik PO, Otto CM, Siemieniuk RA, et al. Transcatheter or surgical aortic valve replacement for patients with severe, symptomatic, aortic stenosis at low to intermediate surgical risk: a clinical practice guideline. BMJ 2016; 354: i5085.

6. Lamontagne F, Meade MO, Hebert PC, et al. Higher versus lower blood pressure targets for vasopressor therapy in shock: a multicentre pilot randomized controlled trial. Intensive Care Med 2016; 42: 542-50.

7. Institute of Medicine Committee on Standards for Developing Trustworthy Clinical Practice Guidelines. In: Graham R, Mancher M, Miller Wolman D, Greenfield S, Steinberg E (Eds). Clinical Practice Guidelines We Can Trust. Washington (DC): The National Academies Press; 2011.

8. Kung J, Miller RR, Mackowiak PA. Failure of clinical practice guidelines to meet institute of medicine standards: two more decades of little, if any, progress. Arch Intern Med 2012; 172: 1628-33.

9. Guyatt GH, Oxman AD, Vist GE, et al. GRADE: an emerging consensus on rating quality of evidence and strength of recommendations. BMJ 2008; 336: 924-6.

10. Hylands $M$, Moller $M H$, Asfar P, et al. A systematic review of vasopressor blood pressure targets in critically ill adults with hypotension. Can J Anesth 2017; 64: this issue. DOI: 10.1007/ s12630-017-0879-Z

11. Hylands M, Kwong J, Toma A, at al. Vasopressor use following traumatic injury: protocol for a systematic review. BMJ Open 2017; 7: e014166.

12. Carrasco-Labra A, Brignardello-Petersen $R$, Santesso $N$, et al. Improving GRADE evidence tables part 1: a randomized trial shows improved understanding of content in summary of findings tables with a new format. J Clin Epidemiol 2016; 74: 7-18.

13. Carrasco-Labra A, Brignardello-Petersen $R$, Santesso $N$, et al. Comparison between the standard and a new alternative format of the summary-of-findings tables in Cochrane review users: study protocol for a randomized controlled trial. Trials 2015; 16: 164.

14. Norris SL, Holmer HK, Ogden LA, Burda BU. Conflict of interest in clinical practice guideline development: a systematic review. PloS one 2011; 6: e25153.

15. Guyatt G, Akl EA, Hirsh J, et al. The vexing problem of guidelines and conflict of interest: a potential solution. Ann Intern Med 2010; 152: 738-41. 